\title{
Carbon Chemistry in Photodissociation Regions
}

\author{
M. Gerin ${ }^{1}$, E. Roueff ${ }^{2}$, J. Le Bourlot ${ }^{2}$, J. Pety ${ }^{3}$, J. R. Goicoechea ${ }^{1}$, \\ D. Teyssier ${ }^{4}$, C. Joblin ${ }^{5}$, A. Abergel ${ }^{6}$, and D. Fossé ${ }^{1}$ \\ ${ }^{1}$ LERMA, Observatoire de Paris and ENS, CNRS UMR8112, \\ 24 Rue Lhomond, 75231 Paris cedex 05, France \\ email: gerin@lra.ens.fr, fosse@lra.ens.fr, javier@lra.ens.fr \\ ${ }^{2}$ LUTH, Observatoire de Paris, CNRS UMR8102, \\ place J. Janssen, 92195 Meudon cedex, France \\ email: evelyne.roueff@obspm.fr, jacques.lebourlot@obspm.fr \\ ${ }^{3}$ J. Pety, IRAM, 300 rue de la piscine, 38406 Grenoble cedex, France \\ email: pety@iram.fr \\ ${ }^{4}$ European Space Astronomy Centre, Villafranca del Castillo, Madrid 28080, Spain \\ email: dteyssier@sciops.esa.int \\ ${ }^{5}$ CESR, CNRS and Univ. Paul Sabatier, 9 Avenue du colonel Roche, 31028 Toulouse, France \\ email: joblin@cesr.fr \\ ${ }^{6}$ Institut d'Astrophysique Spatiale, CNRS et Univ. Paris-Sud, \\ Bât. 121, 91405 Orsay Cedex, France \\ email: alain.abergel@ias.u-psud.fr
}

\begin{abstract}
We present recent results on the carbon chemistry in photodissociation regions. We show that carbon chains and rings $\left(\mathrm{CCH}, \mathrm{c}_{-} \mathrm{C}_{3} \mathrm{H}_{2}\right.$ and $\left.\mathrm{C}_{4} \mathrm{H}\right)$ are tightly spatially correlated with each other, and with the mid-infrared emission due to PAHs ( 7 and $15 \mu \mathrm{m})$, mapped by ISOCAM. Neither the spatial distribution, nor the abundances of these species can be fit by state-of-the-art PDR models, which calls for another production mechanism. We discuss model predictions for carbon clusters and simple hydrocarbons. We show how selected abundance ratios can be used as a diagnostic of the physical conditions. We stress the need for more theoretical and laboratory work on fundamental processes relevant for the interstellar medium, which should be taken into account in the astrochemical models, but whose rates are not known accurately enough.
\end{abstract}

Keywords. astrochemistry — ISM: clouds — ISM: evolution — ISM: molecules — ISM: structure

\section{Introduction}

As carbon is one of the most abundant elements in the interstellar medium, which can bind to other elements in many ways, most of the interstellar and circumstellar molecules contain at least one carbon atom. Table 1 lists the detected interstellar and circumstellar species through September 2005. Carbon species account for $75 \%$ of the 144 detected molecules, and include the most complex and heaviest molecules detected.

Carbon chemistry is also important for understanding the structure and evolution of the interstellar medium (ISM). Carbon monoxide is the most abundant molecule after molecular hydrogen and a widely used tracer of molecular regions, as $\mathrm{H}_{2}$ lines are usually weak and not easily accessible. Carbon chemistry affects the gas cooling processes as $\mathrm{C}^{+}$ and $\mathrm{C}$ fine-structure line emission, together with $\mathrm{CO}$ and $\mathrm{H}_{2} \mathrm{O}$ rotational line emission, 
Table 1. Interstellar and Circumstellar Molecules

\begin{tabular}{|c|c|c|c|c|c|c|}
\hline \multicolumn{7}{|c|}{ Molecular Hydrogen and Related Ions } \\
\hline$\underline{\mathrm{H}_{2}}$ & $\underline{\mathrm{HD}}$ & $\mathrm{H}_{3}^{+}$ & $\mathrm{H}_{2} \mathrm{D}^{+}$ & $\mathrm{D}_{2} \mathrm{H}^{+}$ & & \\
\hline \multicolumn{7}{|c|}{ Carbon Chains and Rings } \\
\hline $\begin{array}{l}\frac{\mathrm{CH}}{\mathrm{C}_{2} \mathrm{H}_{2}} \\
\mathrm{C}_{4} \mathrm{H} \\
\mathrm{C}_{6} \mathrm{H}\end{array}$ & $\begin{array}{l}\mathrm{CH}^{+} \\
l-\mathrm{C}_{3} \mathrm{H} \\
\mathbf{C}_{2} \mathbf{H}_{4} \\
\mathrm{C}_{6} \mathrm{H}_{2}\end{array}$ & $\begin{array}{l}\mathrm{C}_{2} \\
c-\mathrm{C}_{3} \mathrm{H} \\
\mathbf{C}_{5} \\
\mathbf{H C}_{6} \mathbf{H}\end{array}$ & $\begin{array}{l}\mathrm{CH}_{2} \\
\mathrm{CH}_{4} \\
\mathrm{C}_{5} \mathrm{H} \\
\mathbf{C}_{7} \mathbf{H}\end{array}$ & $\begin{array}{l}\frac{\mathrm{CCH}}{\mathrm{C}_{4} ?} \\
l-\mathrm{H}_{2} \mathrm{C}_{4} \\
\mathrm{CH}_{3} \mathrm{C}_{4} \mathrm{H}\end{array}$ & $\begin{array}{l}\mathrm{C}_{3} \\
c-\mathrm{C}_{3} \mathrm{H}_{2} \\
\mathbf{H C} \mathbf{C}_{4} \mathbf{H} \\
\mathrm{C}_{8} \mathrm{H}\end{array}$ & $\begin{array}{l}\mathrm{CH}_{3} \\
l-\mathrm{C}_{3} \mathrm{H}_{2} \\
\mathrm{CH}_{3} \mathrm{CCH} \\
\mathbf{C}_{6} \mathbf{H}_{6}\end{array}$ \\
\hline \multicolumn{7}{|c|}{ Species with $\mathrm{O}$, or $\mathrm{H}$ and $\mathrm{C}$} \\
\hline $\begin{array}{l}\frac{\mathrm{OH}}{\mathrm{HCO}} \\
\mathrm{HCOOH} \\
\mathrm{CH}_{3} \mathrm{CHO} \\
\mathrm{CH}_{3} \mathrm{COOH} \\
\left(\mathrm{CH}_{3}\right)_{2} \mathrm{CO}\end{array}$ & $\begin{array}{l}\frac{\mathrm{H}_{2} \mathrm{O}}{\mathrm{C}_{2} \mathrm{O}} \\
\mathrm{CH}_{2} \mathrm{CO} \\
c-\mathrm{C}_{2} \mathrm{H}_{4} \mathrm{O} \\
\mathrm{CH}_{2} \mathrm{CHOH} \\
\mathrm{CO}\left(\mathrm{CH}_{2} \mathrm{OH}\right)_{2}\end{array}$ & $\begin{array}{l}\mathrm{O}_{2} ? \\
\mathrm{CO}_{2} \\
\mathrm{H}_{2} \mathrm{COH}^{+} \\
\mathrm{CH}_{3} \mathrm{OCHO} \\
\left(\mathrm{CH}_{3}\right)_{2} \mathrm{O}\end{array}$ & $\begin{array}{l}\frac{\mathrm{CO}}{\mathrm{H}_{3} \mathrm{O}^{+}} \\
\frac{\mathrm{CH}_{3} \mathrm{OH}}{\mathrm{CH}_{2} \mathrm{OH}} \mathrm{CHO} \\
\mathrm{CH}_{3} \mathrm{CH}_{2} \mathrm{OH}\end{array}$ & $\begin{array}{l}\mathrm{CO}^{+} \\
\mathrm{HOCO}^{+} \\
\mathrm{CH}_{2} \mathrm{CHO} \\
\mathrm{CH}_{2} \mathrm{CHCHO}\end{array}$ & $\begin{array}{l}\frac{\mathrm{HCO}^{+}}{\mathrm{H}_{2} \mathrm{CO}} \\
\mathrm{HC}_{2} \mathrm{CHO} \\
\mathrm{CH}_{3} \mathrm{CH}_{2-} \\
-\mathrm{CHO}\end{array}$ & $\begin{array}{l}\mathrm{HOC}^{+} \\
\mathrm{C}_{3} \mathrm{O} \\
\mathrm{C}_{5} \mathrm{O} \\
\\
\mathrm{HOCH}_{2-} \\
-\mathrm{CH}_{2} \mathrm{OH}\end{array}$ \\
\hline \multicolumn{7}{|c|}{ Species with $\mathrm{N}$, or $\mathrm{H}$ and $\mathrm{C}$} \\
\hline $\begin{array}{l}\mathrm{N}_{2} \\
\mathrm{NH}_{3} \\
\overline{\mathrm{HC}_{3} \mathrm{~N}} \\
\mathrm{HC}_{3} \mathrm{NH}^{+} \\
\mathrm{HC}_{7} \mathrm{~N}\end{array}$ & $\begin{array}{l}\mathrm{NH} \\
\mathrm{HCNH}^{+} \\
\mathrm{HC}_{2} \mathrm{NC} \\
\mathrm{C}_{5} \mathrm{~N} \\
\mathrm{CH}_{3} \mathrm{C}_{5} \mathrm{~N} ?\end{array}$ & $\begin{array}{l}\frac{\mathrm{CN}}{\mathrm{H}_{2}} \mathrm{CN} \\
\mathrm{NH}_{2} \mathrm{CN} \\
\mathrm{CH}_{3} \mathrm{NH}_{2} \\
\mathrm{HC}_{9} \mathrm{~N}\end{array}$ & $\begin{array}{l}\mathrm{NH}_{2} \\
\mathrm{HCCN} \\
\mathrm{C}_{3} \mathrm{NH} \\
\mathrm{C}_{2} \mathrm{H}_{3} \mathrm{CN} \\
\mathrm{HC}_{11} \mathrm{~N}\end{array}$ & $\begin{array}{l}\frac{\mathrm{HCN}}{\mathrm{C}_{3} \mathrm{~N}} \\
\mathbf{H C}_{4} \mathbf{N} \\
\mathrm{HC}_{5} \mathrm{~N}\end{array}$ & $\begin{array}{l}\frac{\mathrm{HNC}}{\mathrm{CH}_{2} \mathrm{CN}} \\
\frac{\mathrm{CH}_{3} \mathrm{CN}}{\mathrm{CH}_{3} \mathrm{C}_{3} \mathrm{~N}}\end{array}$ & $\begin{array}{l}\frac{\mathrm{N}_{2} \mathrm{H}^{+}}{\mathrm{CH}_{2} \mathrm{NH}} \\
\mathrm{CH}_{3} \mathrm{NC} \\
\mathrm{C}_{2} \mathrm{H}_{5} \mathrm{CN}\end{array}$ \\
\hline \multicolumn{7}{|c|}{ Species with $\mathrm{N}, \mathrm{O}$ or $\mathrm{H}$ and $\mathrm{C}$} \\
\hline NO & $\mathrm{HNO}$ & $\mathrm{N}_{2} \mathrm{O}$ & $\underline{\mathrm{HNCO}}$ & $\mathrm{NH}_{2} \mathrm{CHO}$ & & \\
\hline \multicolumn{7}{|c|}{ Species with S, Si and Other Elements } \\
\hline $\begin{array}{l}\mathrm{SH} \\
\mathrm{SiN} \\
\mathrm{HF} \\
\mathrm{OCS} \\
\mathrm{H}_{2} \mathrm{CS} \\
\mathrm{C}_{5} \mathrm{~S}\end{array}$ & $\begin{array}{l}\frac{\mathrm{CS}}{\mathrm{SiO}} \\
\mathbf{A l F} \\
\mathrm{HCS}^{+} \\
\mathrm{HNCS} \\
\mathrm{FeO}\end{array}$ & $\begin{array}{l}\frac{\mathrm{SO}}{\mathrm{SiS}} \\
\mathbf{C P} \\
c-\mathrm{SiC}_{2} \\
\mathrm{C}_{3} \mathrm{~S} \\
\mathrm{AlNC}\end{array}$ & $\begin{array}{l}\mathrm{SO}^{+} \\
\mathrm{HCl} \\
\mathrm{PN} \\
\mathrm{SiCN} \\
\text { c-SiC }_{3} \\
\mathrm{SiNC}\end{array}$ & $\begin{array}{l}\mathrm{NS} \\
\mathrm{NaCl} \\
\mathrm{H}_{2} \mathrm{~S} \\
\mathrm{NaCN} \\
\mathrm{SiH}_{4} \\
\mathrm{CF}^{+}\end{array}$ & $\begin{array}{l}\mathrm{SiH} \\
\mathrm{AlCl} \\
\mathrm{C}_{2} \mathrm{~S} \\
\mathrm{MgCN} \\
\mathrm{SiC}_{4}\end{array}$ & $\begin{array}{l}\mathrm{SiC} \\
\mathbf{K C l} \\
\mathrm{SO}_{2} \\
\mathrm{MgNC} \mathbf{N C} \\
\mathrm{CH}_{3} \mathrm{SH}\end{array}$ \\
\hline
\end{tabular}

Note: Species detected in circumstellar envelopes only are written in boldface. Species detected in external galaxies are underlined.

and neutral oxygen fine structure line emission are the main gas coolants over a wide range of gas densities and temperatures (see, e.g., Kaufman et al. 1999; Wolfire et al. 2003). Finally, carbonaceous grains and PAHs contain a large fraction of the total carbon. These small solid particles and macro-molecules control the gas heating rate through the photoelectric effect, since the photoelectric efficiency is largest for small particles. Although grains are thought to be formed in circumstellar envelopes, their properties evolve as they travel through the interstellar medium. Various observational pieces of evidence for grain processing have been found (see, e.g., Jones 2005; Dartois et al. 2005). In addition to evolution of the grain material itself, dust grains can either grow, or fragment, in the interstellar medium, depending on their surroundings. Sputtering and fragmentation of carbonaceous grains is likely to release fresh carbon back into the ISM. 

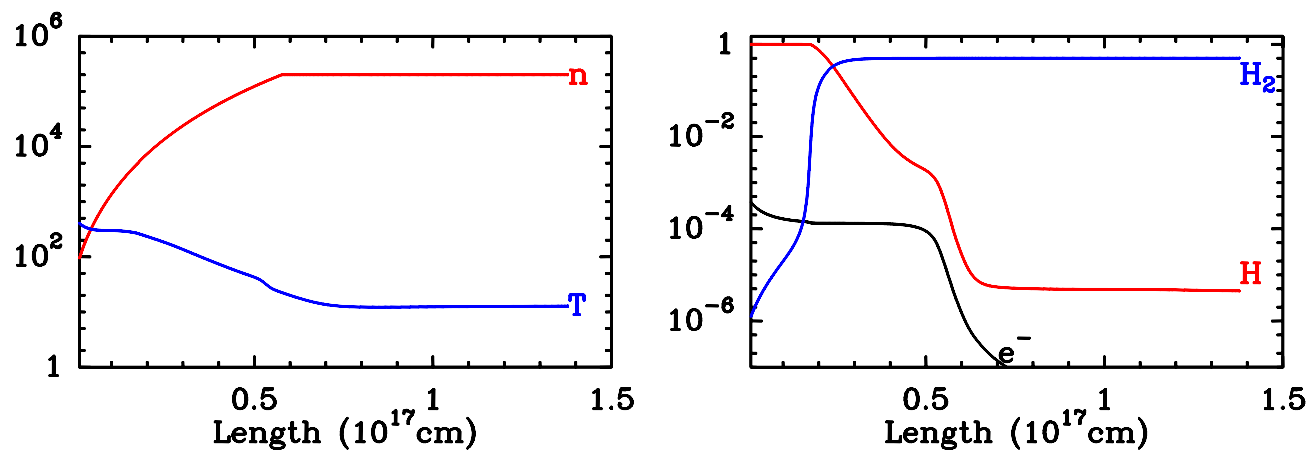

Figure 1. Left: Gas density and temperature for the Horsehead nebula model as a function of distance from the illuminated edge in $\mathrm{cm}$. Right: Abundances of neutral $(\mathrm{H})$ and molecular $\left(\mathrm{H}_{2}\right)$ hydrogen, and electron abundance $\left(\mathrm{e}^{-}\right)$. The FUV radiation field is 100 in Draine's unit.

Photodissociation regions (PDRs) are defined as the warm interfaces, where the gas is molecular but its heating is dominated by FUV photons $(<13.6 \mathrm{eV})$. Such an environment can be found in reflection nebulae - where a massive star illuminates a nearby molecular cloud-but also at the surface of protostellar disks, in circumstellar envelopes and in (proto)planetary nebulae. Because of the enhanced heating, and the efficient cooling due to the moderate gas density, PDRs produce intense continuum and line radiation over a wide range of wavelengths. The intense cooling lines from PDRs are easily detected in external galaxies, and the most distant galaxies become accessible with state-of-the-art instruments (e.g., the detection of the [C II] $158 \mu \mathrm{m}$ line by Maiolino et al. 2005). Therefore PDRs are attractive sources, where the physical and chemical processes relevant for the ISM evolution can be studied in detail, and state-of-the-art models compared with accurate observations.

\section{Carbon Chemistry in the Horsehead Nebula}

\subsection{Observations}

Unsaturated carbon chains and rings are rather abundant molecules in the ISM, and are even more abundant in carbon rich circumstellar envelopes around evolved stars, although these species are extremely reactive in the laboratory. In circumstellar envelopes (most notably the carbon rich star IRC+10216) and in dark clouds, the abundance ratios of these species follow a well-defined pattern with a slow decrease of the abundance as the molecule size increases (Guélin et al. 1977; Teyssier et al. 2004). Until recently, no such systematic studies had been performed for PDRs. Using the IRAM-30m telescope Fuente et al. (2003) observed reactive ions in PDRs and measured high abundances of $\mathrm{HOC}^{+}$ and $\mathrm{CO}^{+}$. They also observed $\mathrm{c}_{-} \mathrm{C}_{3} \mathrm{H}_{2}$ lines in the same sources and concluded that the observed abundance is larger than PDR model predictions. Teyssier et al. (2004) studied the distribution and abundance of selected carbon chains and rings $(\mathrm{CCH}$, the cyclic and linear isomers of $\mathrm{C}_{3} \mathrm{H}$ and $\mathrm{C}_{3} \mathrm{H}_{2}$, and $\mathrm{C}_{4} \mathrm{H}$ ) in three PDRs ( $\rho$ Ophiuchi, IC 63 and the Horsehead nebula). They concluded that the abundance and spatial distribution of these species are not well fitted by PDR models. However, these observations reached a moderate spatial resolution $\left(\sim 20^{\prime \prime}\right)$, which did not allow the authors to resolve the steep abundance gradients predicted by the PDR models.

The Horsehead nebula was selected for a follow-up study at higher spatial resolution, using the IRAM Plateau de Bure interferometer. The Horsehead nebula is particularly 


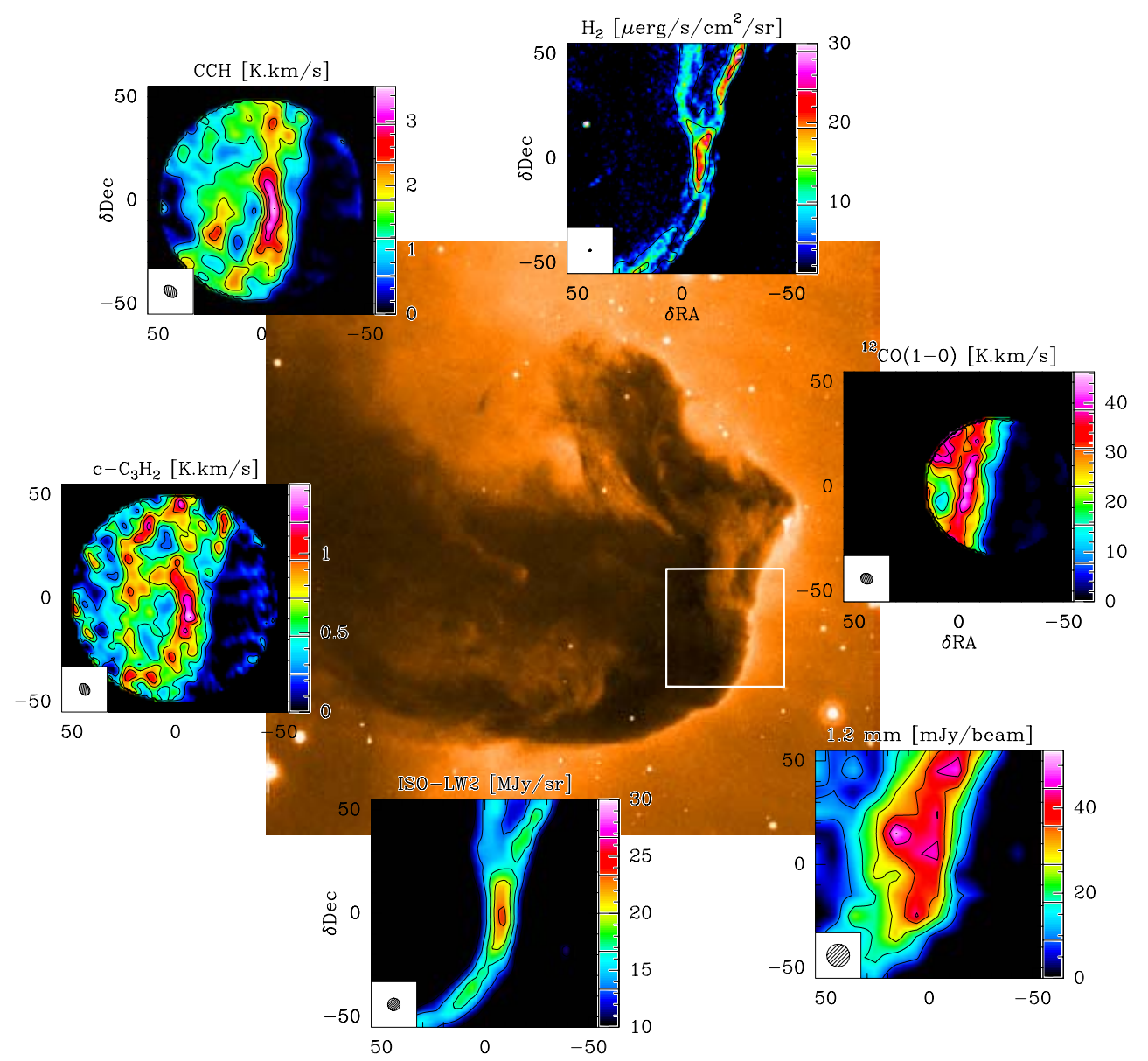

Figure 2. Summary of observations of the Horsehead nebula in LDN1630 from Pety et al. (2005) and Habart et al. (2005). The background image is taken from the ESO archive. The white square indicates the observed field of view of the IRAM Plateau de Bure interferometer. The $\mathrm{CCH}$ (top left), $\mathrm{c}-\mathrm{C}_{3} \mathrm{H}_{2}$ (left), and ${ }^{12} \mathrm{CO}(1-0)$ (right) maps have been obtained with this facility. The PdBI data have been combined with short spacings from the IRAM-30m telescope to recover all spatial frequencies. The $\mathrm{H}_{2} \mathrm{v}=1-0 \mathrm{~S}(1)$ image (top) is from ESO-NTT, the $1.2 \mathrm{~mm}$ image (bottom right) from MAMBO on the IRAM-30m telescope, and the ISO-LW2 image (bottom) has been taken with ISOCAM (Abergel et al. 2003). Note the similarity of the CCH and $\mathrm{c}_{-} \mathrm{C}_{3} \mathrm{H}_{2}$ maps with the ISO-LW2 image, while the $\mathrm{CO}(1-0)$ and dust maps look different.

well suited as a template PDR, as it is viewed nearly edge-on (Habart et al. 2005 for a thorough discussion on the viewing geometry) and is nearby. At a distance of $400 \mathrm{pc}$, $1^{\prime \prime}$ corresponds to a linear size of $6.0 \times 10^{15} \mathrm{~cm}$, or $0.002 \mathrm{pc}$. The Horsehead nebula is illuminated by the O9.5 star $\sigma$ Ori, $\sim 3.5$ pc away, resulting in a far UV radiation field $\mathrm{G}_{0} \sim 60$ times the mean interstellar radiation field, as determined by Draine (1978). As shown by Habart et al. (2005) and Pety et al. (2005), the gas density profile is well constrained by the infrared maps of the $\mathrm{H}_{2}$ rovibrational emission, $v=1-0 \mathrm{~S}(1)$ at $2.1 \mu \mathrm{m}$, and of the PAH emission near $7 \mu \mathrm{m}$. The best-fit density profile rises steeply (in $\sim 10^{\prime \prime}$ ) from $n_{\mathrm{H}} \sim 10^{4} \mathrm{~cm}^{-3}$ to $n_{\mathrm{H}} \sim 10^{5} \mathrm{~cm}^{-3}$, the resulting model being almost isobaric (constant pressure). Figure 1 presents the adopted model.

A summary of the maps is shown in Figure 2. As discussed extensively by Pety et al. (2005), the spatial distribution of hydrocarbons is very similar to the PAH emission, 

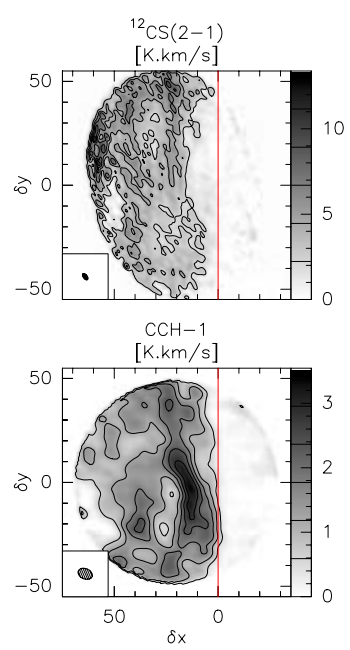
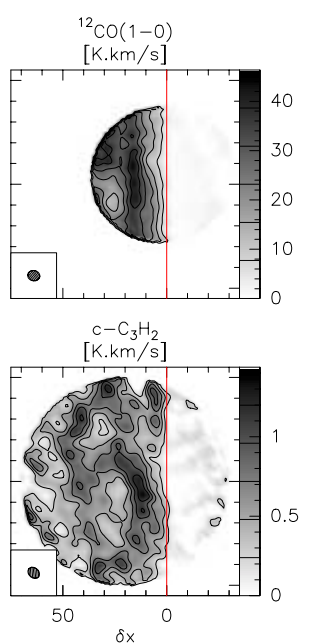

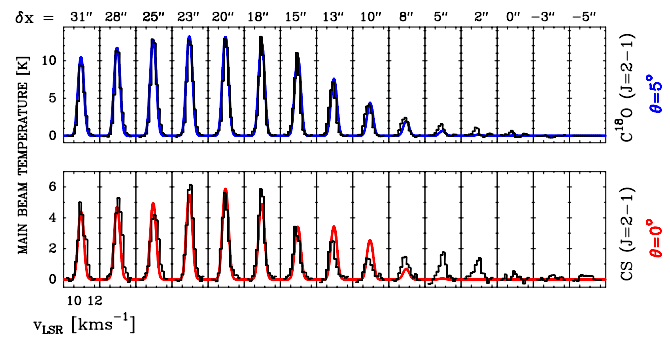

Figure 3. Left: PdBI Maps of the $\mathrm{CS}(2-1), \mathrm{CO}(1-0), \mathrm{CCH}(1-0)$ and $\mathrm{c}_{-} \mathrm{C}_{3} \mathrm{H}_{2}\left(2_{1,2}-1_{0,1}\right)$ lines. The maps have been rotated by $14^{\circ}$ in to ease the comparison with 1-D models. Right: Comparison of observed and modelled $\mathrm{C}^{18} \mathrm{O}$ and CS spectra (from Goicoechea et al. 2005).

and different from $\mathrm{CO}$ and other species. Both the spatial distribution and the molecule abundance could not be fitted by PDR models, even by trying different chemical networks or small modifications of the source geometry and density structure. Because the molecule destruction is dominated by photodissociation in the external layers where the carbon chains are most abundant, detecting species with high abundance implies that their formation is efficient. Pety et al. (2005) reached similar conclusions as Fuente et al. (2003) and Rizzo et al. (2003, 2005), namely that the PAHs responsible for the mid-infrared emission features play a role in the formation of small carbon chains and rings. The most likely explanation is that, when these particles are released from larger structures (Rapacioli et al. 2005; Jones 2005), a fraction of these PAHs fragment and feed the gas with small carbon clusters and molecules.

\subsection{Carbon Molecules and Grains}

Indeed, much observational evidence has now been presented on the evolution of carbonaceous material in the interstellar medium. For instance, Rapacioli et al. (2005) have isolated three characteristic spectra in the reflection nebula NGC 7023, which neatly trace the evolution of PAHs as a function of the distance to the illuminating star. Vijh et al. (2005) discuss the spatial distribution of the recently discovered blue luminescence and also conclude that the carriers are liberated in situ, from larger carbonaceous structures.

While PDR models take into account the role of PAHs in the ionization balance, the influence of PAHs on the carbon chemistry has not been studied yet, because of the lack of basic information. Le Page et al. (2001, 2003) discuss the processes governing the size distribution, ionization balance and hydrogen coverage of PAHs in the ISM. The destruction of PAHs is limited to photodissociation because they do not include possible reactions with species other than hydrogen. Data on reaction rates between oxygen, carbon or nitrogen and PAHs are critically needed to assess possible growth paths of carbon species in the ISM.

\subsection{Further Comparison with Observations}

While the above analysis is based on comparisons of molecular abundances, it is now possible to perform a more accurate comparison between models and observations, 

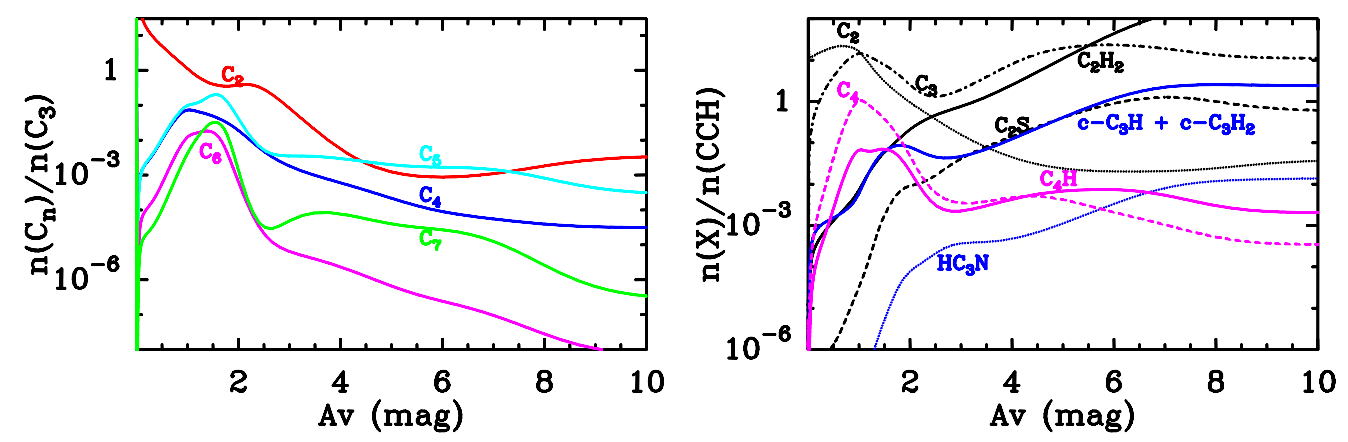

Figure 4. Prediction of the abundance ratio of carbon clusters (right) and other hydrocarbons (left) compared to $\mathrm{C}_{3}$ and $\mathrm{CCH}$ respectively, for physical conditions of the Horsehead nebula.

using the full power of the high spectral resolution provided by millimeter observations. Figure 3 shows how well observed spectra can be fitted by combined PDR and MonteCarlo radiative transfer models. A very small inclination along the line of sight $\left(6^{\circ}\right)$ can be identified when performing such detailed modelling work.

\subsection{Carbon Budget}

As shown in Fig. $4, \mathrm{C}_{2}$ is predicted to be abundant at low $A_{V}$, while $\mathrm{C}_{3}$ and heavier carbon clusters are predicted to be most abundant at moderate extinctions, $A_{V} \sim 1-$ 3 mag. Saturated species, such as acetylene, $\mathrm{C}_{2} \mathrm{H}_{2}$, participate in the carbon budget at higher $A_{V}$ 's. Though the carbon chemistry is still not well understood, these conclusions are qualitatively in agreement with the observational data. Carbon clusters $\left(\mathrm{C}_{2}, \mathrm{C}_{3}\right.$ and maybe even $\mathrm{C}_{4}$ ) have been widely observed in the ISM (Oka et al. 2003; Cernicharo et al. 2002), with a tight correlation between $\mathrm{C}_{2}$ and $\mathrm{C}_{3}$ abundances. The low abundance of both $\mathrm{HC}_{3} \mathrm{~N}$ and $\mathrm{CH}_{3} \mathrm{CCH}$ in the Horsehead nebula PDR (Teyssier et al. 2004) is also consistent with model predictions.

\section{Carbon Chemistry as a Tracer of Gas Properties}

Chemical models show that interstellar molecular abundances are sensitive to the gas physical conditions, such as the density, temperature, but also the electron abundance. This important parameter for the gas physics cannot be measured directly, as the main carriers of the charges cannot be observed. However, a few molecular abundance ratios have been shown to scale with the electron abundance. In dense and shielded regions, the degree of deuterium fractionation of molecular ions $\left(\mathrm{HCO}^{+}, \mathrm{N}_{2} \mathrm{H}^{+}\right.$, etc.) can be used to measure the electron abundance (Guélin et al. 1982; Caselli et al. 1999). Fossé et al. (2001) have showed that the $\left[\mathrm{c}-\mathrm{C}_{3} \mathrm{H}_{2}\right] /\left[1-\mathrm{C}_{3} \mathrm{H}_{2}\right]$ abundance ratio is sensitive to the electron abundance in the dark cloud TMC-1. Teyssier et al. (2005) have presented observational evidence for a similar behaviour in the Horsehead PDR. Figure 5 presents model predictions of abundance ratios as a function of the electron density. While $[\mathrm{HCN}] /[\mathrm{HNC}]$ is not sensitive to the electron abundance, the ratio of $\mathrm{C}_{3} \mathrm{H}_{2}$ and $\mathrm{C}_{3} \mathrm{H}$ isomers can indeed be used to estimate the fractional ionization. As suggested by Sternberg (2005, this volume), $[\mathrm{CN}] /[\mathrm{HCN}]$ can also be used for probing the electron abundance. $[\mathrm{CN}] /[\mathrm{HCN}]$ gradients have been observed in the Orion Bar and NGC 7023 by A. Fuente and co-workers (e.g., Fuente et al. 1993), though the spatial resolution provided by a single dish telescope was 


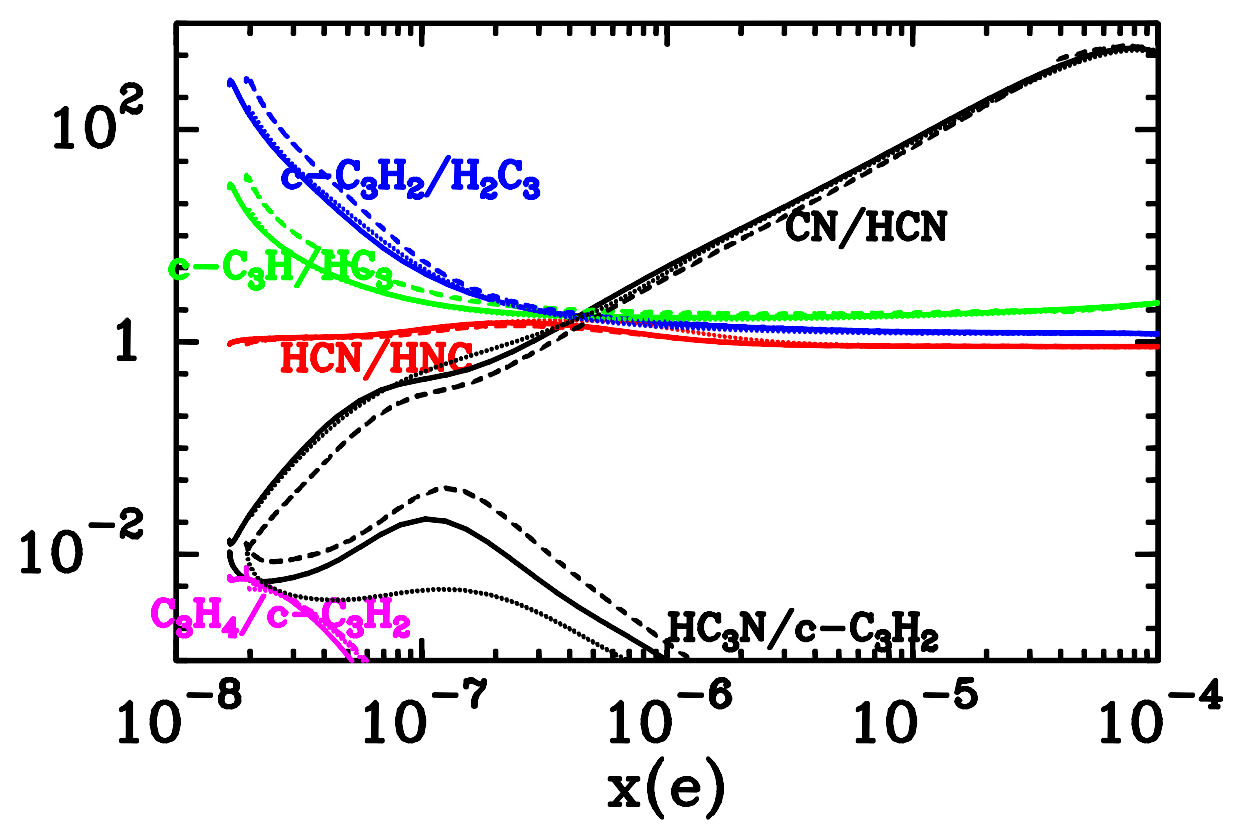

Figure 5. Molecular abundance ratios as a function of electron density for conditions suited to the Horsehead nebula. The radiation field is $G_{0}=100$. The full line corresponds to a varying gas density, with a complete thermal balance. The dashed line corresponds to a model using the same density profile, but with a minimum temperature set to $30 \mathrm{~K}$, as used by Goicoechea et al. (2005) to fit the $\mathrm{CS}$ and $\mathrm{C}^{18} \mathrm{O}$ data. The dotted line corresponds to a model with a constant gas density of $n_{\mathrm{H}}=10^{5} \mathrm{~cm}^{-3}$. High electron abundances are found in the warm outer layers, while the low values correspond to the dense, well shielded regions (see Fig. 1).

not quite sufficient. Higher spatial resolution maps, which can now be obtained with millimeter interferometers, can be used to constrain the models accurately.

\section{Conclusions}

With the current generation of instruments, and even more with the upcoming generation, excellent data can be obtained, which will allow us to probe interstellar chemistry accurately. State-of-the-art chemical models can now be combined with sophisticated radiative transfer codes to obtain accurate predictions of the line and continuum emission, which can be compared with the observed data. This method requires a simple source geometry (an inaccurate geometry can lead to important differences in line predictions and invalid conclusions). PDRs will remain template sources where physical processes, which operate on a wide range of physical conditions, can be studied with the greatest detail. Carbon chains and rings, and carbon clusters, are promising probes of the interstellar chemistry as they can be used for studying the ionization balance, the relationship between dust grains, macromolecules and gas phase species, as well as the formation routes of complex molecules.

However, PDR models still need improvement. In the coming years, advances are expected on the following aspects:

- Many parameters which are used in current models suffer from large uncertainties. Chemical reaction rates, such as those governing the sulfur chemistry for instance, are not known to the required accuracy over the temperature range relevant for interstellar chemistry. 
- Although PDR models solve self-consistently for the gas chemistry and thermal balance, the predicted temperatures are too low at moderate extinction. This old problem has important consequences, as the predicted intensities of millimeter rotational lines are strongly dependent on the assumed gas kinetic temperature. Hence molecular abundances deduced from those lines can be affected by an error in the gas temperature determination.

- Observations of both gas-phase and solid-phase species point to the necessity of including a tight coupling of the dust and gas phase chemistry in the models. This requires, however, the knowledge of reaction rates for reactions involving abundant ions and atoms and PAHs, for instance. Laboratory studies are underway, as shown by posters presented at this conference (Betts et al. 2005; Joblin et al. 2005).

- Most PDR models solve the gas chemical abundances at steady state, in order to be able to correctly treat the FUV radiative transfer and $\mathrm{H}_{2}$ photodissociation and ro-vibrational excitation. However, this hypothesis is not completely valid, as PDR are usually bordered by an $\mathrm{H}$ II region (associated with the illuminating star), which could be in expansion. Further developments could include a combined treatment of the ionized, neutral and molecular gas, as presented by R. Ferland and co-workers for the CLOUDY model (Ferland et al. 2005). Work aiming at understanding the temporal evolution of a PDR (see e.g; Morata \& Herbst 2005) should also be pursued. Finally dynamical models, such as those presented by Hosokawa \& Inutsuka (2005), provide a complementary view of the evolution of the gas density structure.

\section{Acknowledgements}

We thank the organizers for a very nice conference in beautiful Asilomar. We would like to acknowledge funding by the CNRS/INSU Programme PCMI and CNFA.

\section{References}

Abergel, A., Teyssier, D., Bernard, J.P., et al. 2003, A\& A 410, 577

Betts, N., Bierbaum, V.M., \& Snow, T.P. 2005, IAUS 231, poster

Caselli, P., Walmsley, C.M., Tafalla, M., Dore, L., \& Myers, P.C. 1999, Ap. J. 523, L165

Cernicharo, J., Goicoechea, J.R., \& Benilan, Y. 2002, Ap. J. 580, L157

Dartois, E., Muñoz-Caro, G.M., Deboffle, D., Montagnac, G., \& D'Hendecourt, L. 2005, A\&A 432,895

Draine, B.T. 1978, Ap. J. Suppl. 36, 595

Ferland, G.J., Abel, N., Elwert, T., Porter, R., \& Shaw, G. 2005, IAUS 231, poster

Fossé, D., Cernicharo, J., Gerin, M., \& Cox, P. 2001, Ap. J. 552, 168

Fuente, A., Martin-Pintado, J., Cernicharo, J., \& Bachiller, R. 1993, A $\&$ A 276, 473

Fuente, A., Rodriguez-Franco, A., Garcia-Burillo, S., Martin-Pintado, J., \& Black, J.H. 2003 $A \mathscr{G} A, 406,899$

Goicoechea, J.R., Pety, J., Gerin, M., Roueff, E., Teyssier, D., Abergel, A., Habart, E., \& Joblin, C. 2005, IAUS 231, poster

Guélin, M., Langer, W.D., \& Wilson, R.W. 1982, A\&A 107, 107

Guélin, M., Cernicharo, J., Travers, M.J. et al. 1997 A $\& A$ 317, L1

Habart, E., Abergel, A., Walmsley, C.M., Teyssier, D., \& Pety, J. 2005, A\&\&A 437, 177

Hosokawa, T. \& Inutsuka, S. 2005, Ap. J. 623, 917

Joblin, C., Abergel, A., Bernard, J.-P., et al. 2005, IAUS 231, poster

Jones, A. 2005, IAUS 231, poster

Kaufman, M.J., Wolfire, M.G., Hollenbach, D.J., \& Luhman, M.L. 1999, Ap. J. 527, 795

Le Page, V., Snow, T.P., \& Bierbaum, V.M. 2001, Ap. J. Suppl. 132, 233

Le Page, V., Snow, T.P., \& Bierbaum, V.M. 2003, Ap. J. 584, 316

Maiolino, R., Cox, P., Caselli, P., et al. 2005, A\&A, in press 
Morata, O. \& Herbst, E. 2005, IAUS 231, poster

Oka, T., Thorburn, J.A., McCall, B.J. et al. 2003, Ap. J. 582, 823

Pety, J., Teyssier, D., Fossé, D., Gerin, M., Roueff, E., Abergel, A., Habart, E., \& Cernicharo, J. 2005, A\&A 435, 885

Rapacioli, M., Joblin, C., \& Boissel, P. 2005, A\&A 429, 193

Rizzo, J.R., Fuente, A., Rodriguez-Franco, A., \& Garca-Burillo, S. 2003, Ap. J. 597, L153

Rizzo, J.R., Fuente, A., \& Garcia-Burillo, S. 2005, Ap. J., in press

Sternberg, A. 2005, this volume

Teyssier, D., Fossé, D., Gerin, M., Pety, J., Abergel, A., \& Roueff, E. 2004, A\&A 417, 135

Teyssier, D., Hily-Blant, P., Gerin, M., Cernicharo, J., Roueff, E., \& Pety, J. 2005, in The dusty and molecular universe: a prelude to Herschel and ALMA, ed. A. Wilson (ESA SP-577), p. 423

Vijh, U.P., Witt, A.N., \& Gordon, K.D. 2005, Ap. J., in press

Wolfire, M.G., McKee, C.F., Hollenbach, D., \& Tielens, A.G.G.M. 2003, Ap. J. 587, 278

\section{Discussion}

PAPADOPOUlOs: It has been 20 years since the first PDR models were published. Since then the "sandwich" distribution of $\mathrm{C}^{+} / \mathrm{C}$ I/CO at the edges, $A_{v} \sim 1-3$, of clouds has failed to show up anywhere in the sky. So why should we believe/trust any other predicted distribution of species coming out of such models?

GERIN: First, the model fares well for other species: CO, $\mathrm{C}^{18} \mathrm{O}$, CS for instance. An accurate fit needs to consider the cloud geometry: the predictions for an infinite edge-on slab need to be tailored for a finite slab of gas, including two surfaces along the line of sight which contribute some of the emission for $\mathrm{C}$ and ${ }^{12} \mathrm{CO}$. A careful understanding of the source geometry and structure is mandatory before assessing model success and failures. Of course, the question of the atomic carbon abundance and spatial distribution is probably not totally understood yet.

GUÉLIN: What about desorption from dust grains in PDRs?

GERIN: Desorption from dust grains could be considered, but it is most relevant for species which are abundant in the mantles $\left(\mathrm{H}_{2} \mathrm{O}, \mathrm{CH}_{3} \mathrm{OH}, \mathrm{H}_{2} \mathrm{CO}\right.$, etc. $)$, and not so much for the hydrocarbons, which we study. 


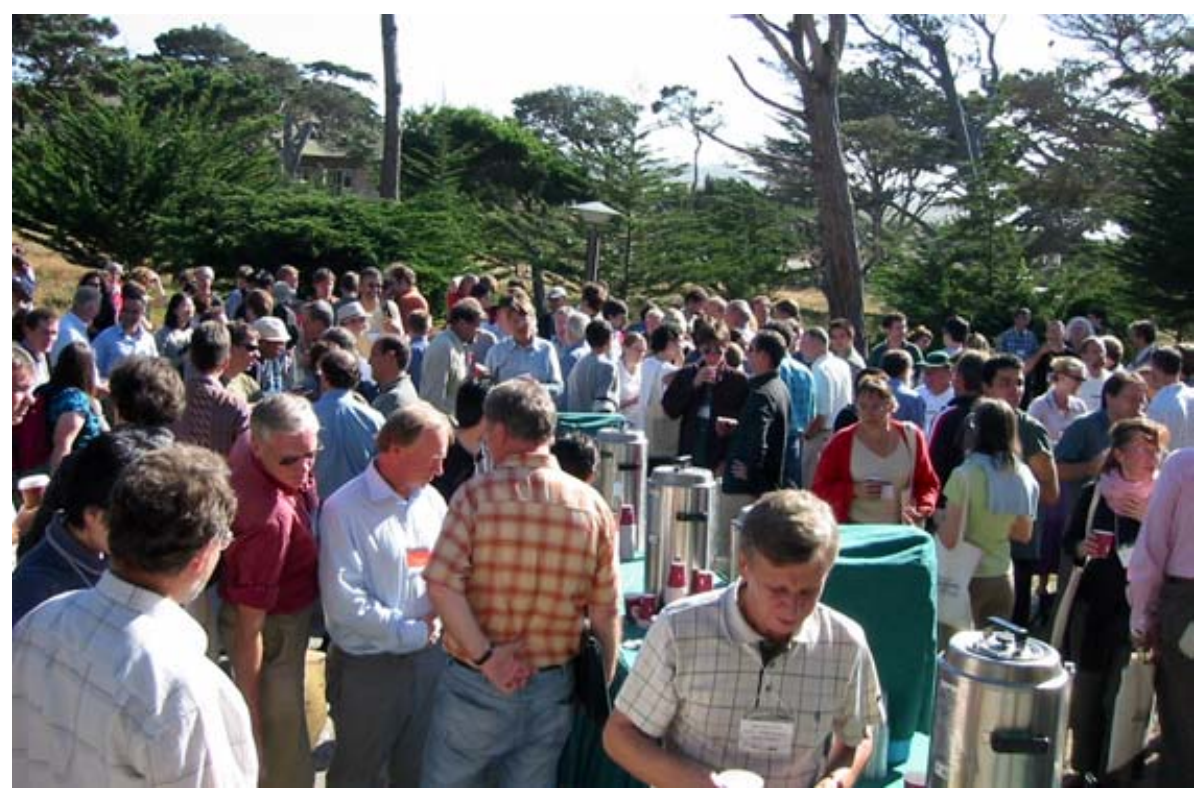

Photo: E. van Dishoeck 